\title{
Experimental and Simulation Analysis for the Impact of a Two-Link Chain with Granular Matter
}

\author{
Eliza A. Banu and Dan B. Marghitu \\ Mechanical Engineering Department, Auburn University, Auburn, AL 36849, USA \\ Correspondence should be addressed to Eliza A. Banu; banueli@auburn.edu
}

Received 30 October 2014; Revised 22 May 2015; Accepted 2 June 2015

Academic Editor: Sundararajan Natarajan

Copyright (C) 2015 E. A. Banu and D. B. Marghitu. This is an open access article distributed under the Creative Commons Attribution License, which permits unrestricted use, distribution, and reproduction in any medium, provided the original work is properly cited.

\begin{abstract}
The resistance force of the granular matter is modeled as a linear superposition of a static (quadratic depth-dependent) resistance force and a dynamic (quadratic velocity-dependent) frictional force. The impact is defined from the moment the end point of the system comes in contact with the granular matter surface until the vertical linear velocity of the end point is zero. The variables of interest are the final depth at the end of the penetration phase and the stopping time. The results for a two-link kinematic chain with two points of contact were compared to the results obtained by applying the resistance force formulation developed to corresponding CAD simulation models. The results revealed that the final displacement increases with initial velocity, while the stopping time decreases. The sensitivity to the initial velocity was studied and an improvement to the resistance force formulated as a result. A series of expressions are proposed for the resistance force coefficients.
\end{abstract}

\section{Introduction}

Impact with granular matter has been of great interest to researchers for decades, mainly because although the material is a conglomeration of discrete solids, it behaves as a fluid until a solid-like behavior becomes established. As a result, a resistance force that would account for these characteristics during impact has been difficult to model. The dynamics associated with the motion and interaction of rigid bodies with Newtonian fluids is a classical problem in fluid mechanics. Granular materials are collections of polydisperse grains and exhibit complex behaviors. This means that the rheology of the medium is comparable to that of a solid under a critical shear stress, although it performs like a fluid above it. Granular materials have been studied for potential applications in multiple areas. In earth science, for example, problems such as avalanches involve the flow of granular matter $[1,2]$ and a better understanding of their behavior would greatly facilitate those seeking to model the effects of earthquakes [3], meteorite impact cratering, and low-speed impact cratering [4-6]. Industrial processes such as mixing, stirring, and drilling would also benefit $[7,8]$. To appreciate the larger perspective that is involved in these applications, the impact of an object with granular matter is being explored through experiment, simulation, and theory. However, because of the solid-fluid-like behavior of the granular matter, a great deal remains to be explored, especially from a theoretical perspective. The focus of much of the current research on the impact of a rigid body on granular matter is on describing the force experienced due to the reorganization of the grains that are opposing the motion of the intruder. A good understanding of these forces can aid in the design of tools and the design and control of robots designed to maneuver in granular environments. Due to the lack of a universal accepted theoretical model, experiments have generally sought to demonstrate the variation of the drag force in terms of a number of different parameters: impact velocity [9], the diameter of the penetrating object $[1,9]$, the packing volume [10], and the shape [11] of the penetrating object, for example, cylinders $[7,9,12-14]$ or spheres $[3,15,16]$. Others have adopted a slightly different approach by performing their analysis using discrete element methods (DEM) for the same purpose $[1,17,18]$.

The penetration of granular matter at high speeds and low speeds has been studied in recent years $[5,6,16,19$, 20]. In particular, studies of impact cratering have looked 
at parameters such as the depth and size of the crater, as well as how the form of the crater is affected by the initial impact conditions of the impacting rigid body. To study the motion of a sphere through granular matter resistance force models that treated the problem as the sum of a velocitydependent force and a static resistance force which is depthdependent were created [5]. For example, Ambroso et al. [19] suggested that the stopping time of a projectile in granular matter depends on the geometry and density of the rigid body and the initial impact velocity.

Lee and Marghitu [21] extended the work of Katsuragi and Durian [4] to provide the first mathematical model for an oblique impact. They demonstrated that for an oblique impact of a compound pendulum the stopping time in the granular matter increases with decreasing initial impact velocity using a force model that consists of a linear superposition of the static resistance force and drag force.

The focus of this study is on the oblique impact of rigid bodies. Research shows that the study of the impact of rigid bodies with granular matter is potentially of great significance. As yet, to the best of our knowledge there has been no comprehensive universally accepted expression for the penetration resistive force of a rigid projectile into a granular medium. The most important aspect of this analysis is therefore to develop an analytical expression for the resistant force that is valid for low-speed impact both vertically and at an angle. Researchers have analyzed the impact of spherical and cylindrical shaped rigid bodies through experiments and numerical simulation. It was concluded that the drag force [16] should include a quadratic velocity-dependent term $[4,5]$ and depend on the depth of penetration $[2,7,9,11$, $22,23]$. Research has also shown that the resistant force and depth of penetration for plunging rigid bodies into granular matter depend on other parameters as well. The resistant force during impact scales with impact velocity [16], diameter of the impacting object $[2,9,24]$, and packing fraction $[2,10$, 25]. Only a few studies have been reported for oblique angle impact [21] but have shown that the impact angle has an influence on the value of the resistant force $[1,13,18,26,27]$.

The first objective of this paper is to examine the characteristics of the resistance force presented above and to develop a model that takes into account the granular properties of the medium. Until now, the coefficients employed in these expressions have been determined experimentally. Instead a series of mathematical expressions will be proposed and then validated by comparing the simulation results for depth and stopping time for double impact points at different angles with the experimental results.

A second purpose is to validate the use of a CAD software package in simulating the impact with granular matter by using an equation model that will reduce computational time in comparison to other numerical models and discrete element methods. The results of this study will be of a particular value in robotic design, prosthetics, and biomechanics.

This study is of great importance and a set of mathematical expressions for estimating the values of the scaling that should be used is proposed for each component of the resistance force. The force model presented here shows that the two components are not independent of each other and should thus not be treated separately. The depth depends on impact velocity; therefore the static force will be influenced by the dynamic force.

\section{Mathematical Model}

The first mathematical model for the impact of an object with granular matter at a specific angle was proposed by Lee and Marghitu [21] based on the force model proposed by Tsimring and Volfson [5] and verified experimentally for a sphere by Katsuragi and Durian [4]. The resistance force proposed takes into account both the rate-dependent force (dynamic frictional force) and the displacement-dependent force (static resistance force):

$$
F_{r}=F_{d}(v)+F_{s}(y) .
$$

The dynamic force $F_{d}(v)$ is due to the velocity, $v$, of the intruder in the granular matter, which is made possible by the fluid-like characteristics of the material, up to the point at which the granular material begins to act like a solid surrounding the object, stopping its motion. Hence, the expression for this can take the same form as that for the drag force: $F_{d}=b v$. However, it has been shown that the rate-dependent force is proportional to the velocity squared $[9,25]: F_{d}=0.5 \rho A C_{d} v^{2}$, where $\rho$ is the density of the medium, $A$ is the reference area, and $C_{d}$ is the drag coefficient. The dynamic force used in this work is

$$
\mathbf{F}_{d}=-\frac{\mathbf{v}}{|\mathbf{v}|} \eta_{d} A_{r} \rho_{g} \mathbf{v} \cdot \mathbf{v} .
$$

The force is opposing the motion of the penetrating body; therefore, the force expression includes the term $-\mathbf{v} /|\mathbf{v}|$. The term $\eta_{d}$ is a constant and $A_{r}$ is the reference area.

The static force, $F_{s}$, in (1) represents the force that appears due to the hydrostatic pressure [10] and is based primarily on the reorganization of the particles of matter and grain friction. Thus, this force depends on the material properties, the packing of the matter $[2,17,25]$, gravitation, and size and shape of the penetrating object. This will tend to lead to the stress on one side to increasing with depth according to the scaling factor in one direction and according to a power law for depth in another direction.

The horizontal static force increases with the granular pressure $[9,15,24,28]$ that is acting on the surface of the body in the horizontal direction. From experiments conducted [9] at very low speeds of $v_{0}<5 \mathrm{~mm} / \mathrm{s}$, the yield force is of the form $F=\eta g \rho H^{2} d_{c}$, where $\eta$ is a constant that depends on the media properties, $g$ is the gravitation acceleration, $H$ is the immersed dimension of the intruder, and $d_{c}$ is the diameter of the object. The direction in which the force acts will be opposite to the normal velocity $\mathbf{v}_{h}$ of the center of mass of the immersed volume, $V$, and the immersed length of the rigid body is given by the displacement in the granular matter of the tip of the cylinder, $d_{y_{T}}$. Therefore the generalized horizontal static force is

$$
\mathbf{F}_{\mathrm{sh}}=-\frac{\mathbf{v}_{h}}{\left|\mathbf{v}_{h}\right|} \eta_{h} g \rho_{g} d_{c} d_{y_{T}}^{2} .
$$


The vertical component of the static force represents the resistant force of the granular medium to the immersion of the intruder in the vertical direction. Experiments conducted for vertical impact $[3,15,23]$ revealed a nonlinear dependency of the force on the depth of penetration and attempted to formulate a power law to describe this force. The power law form was proven to arise due to the dispersed quality of the grain size of the granular matter found in nature, so that the force chain structure between the grains and the grain intruder exhibits a homogenous distribution. Thus, the expression for the vertical static force is

$$
\mathbf{F}_{\mathrm{sv}}=-\frac{\mathbf{v}_{v}}{\left|\mathbf{v}_{v}\right|} \eta_{v}\left(\frac{d_{y_{T}}}{L}\right)^{\lambda} g \rho_{g} V
$$

where $L$ is the length of the rigid body and $\mathbf{v}_{v}$ is the vertical term of the velocity of the center of mass of the immersed volume, $V$.

For a two-link kinematic chain with two contact points, (2), (3), and (4) can be applied for each contact point. The dynamic friction force and static resistance force have the same point of application. The point of application is considered to be the centroid of the immersed volume. For a Cartesian reference frame the terms of the resistance force become

$$
\begin{aligned}
\mathbf{F}_{\mathbf{d}} & =-\frac{\mathbf{v}_{E}}{\left|\mathbf{v}_{E}\right|} \eta_{d} \rho_{g} A_{r} \mathbf{v}_{E} \cdot \mathbf{v}_{E}=-\mathbf{v}_{E} \eta_{d} \rho_{g} A_{r}\left|\mathbf{v}_{E}\right|, \\
\mathbf{F}_{\mathrm{sh}} & =-\frac{\mathbf{v}_{E_{x}}}{\left|\mathbf{v}_{E_{x}}\right|} \eta_{h} g \rho_{g} d_{y_{T}}^{2} d_{c} \\
& =\left[-\operatorname{sign}\left(v_{E_{x}}\right) \eta_{h} g \rho_{g} d_{y_{T}}^{2} d_{c}\right] \boldsymbol{\imath}, \\
\mathbf{F}_{\mathrm{sv}} & =-\frac{\mathbf{v}_{E_{y}}}{\left|\mathbf{v}_{E_{y}}\right|} \eta_{v}\left(\frac{d_{y_{T}}}{L}\right)^{\lambda} g \rho_{g} V \\
& =\left[-\operatorname{sign}\left(v_{E_{y}}\right) \eta_{v}\left(\frac{d_{y_{T}}}{L}\right)^{\lambda} g \rho_{g} V\right] \boldsymbol{j},
\end{aligned}
$$

where $L$ is the lateral dimension of the link, $d_{c}$ is the diameter, and $\rho_{g}$ is the density of the granular medium. Because of the proven dependency of the resistance force of the packing fraction of the granular matter, $\rho_{g}$ is the density of the medium and the packing fraction is factored into the value of $\rho_{g}$. The velocity $\mathbf{v}_{E}$ is the linear velocity vector of the centroid of the immersed volume, with $\mathbf{v}_{E_{x}}$ being the horizontal component of the velocity $\mathbf{v}_{E}$ and $\mathbf{v}_{E_{y}}$ the vertical component of the velocity of the centroid $\mathbf{v}_{E}$.

The velocity of point $E$, as shown in Figure 1, can be expressed in terms of the velocity of the end point $T, v_{T}$, angle of impact, $q$, and angular velocity of the bar, $\omega$ :

$$
\mathbf{v}_{E}=\mathbf{v}_{T}+\boldsymbol{\omega} \times\left(\mathbf{r}_{E}-\mathbf{r}_{T}\right),
$$

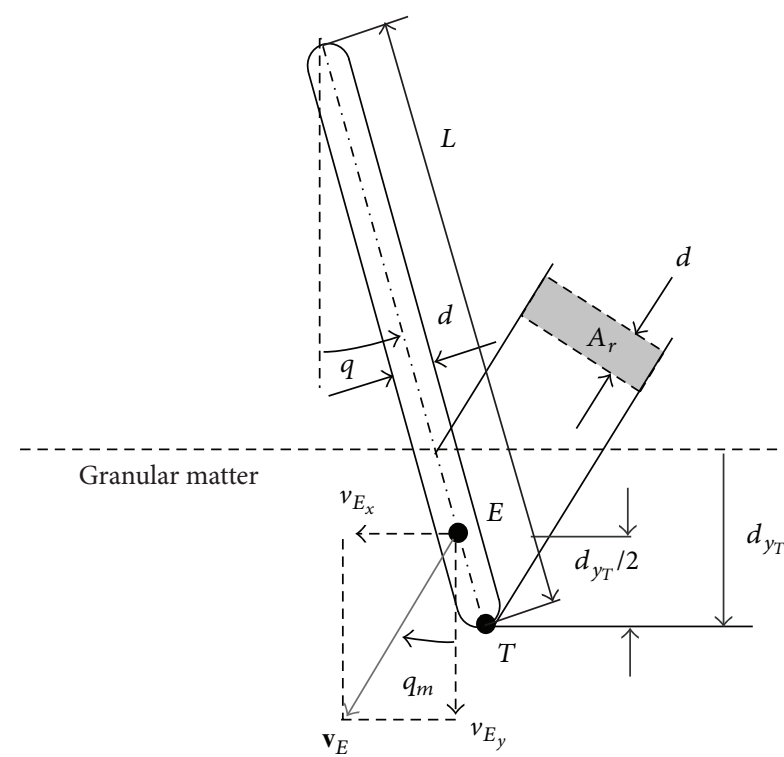

FIGURE 1: The reference area and the physical parameters of the free link.

where $\mathbf{r}_{E}$ and $\mathbf{r}_{T}$ are the position vectors of points $E$ and $T$ :

$$
\begin{aligned}
& \mathbf{r}_{T}=d_{y_{T}} \boldsymbol{\jmath}, \\
& \mathbf{r}_{E}=\left(\frac{d_{y_{T}}}{2} \cdot \tan (q)\right) \boldsymbol{\imath}+\frac{d_{y_{T}}}{2} \boldsymbol{\jmath} .
\end{aligned}
$$

Then

$$
\begin{aligned}
& \mathbf{r}_{E}-\mathbf{r}_{T}=\left(\frac{d_{y_{T}}}{2} \cdot \tan (q)\right) \boldsymbol{\imath}+\frac{d_{y_{T}}}{2} \boldsymbol{\jmath}-d_{y_{T}} \boldsymbol{j}, \\
& \mathbf{r}_{E}-\mathbf{r}_{T}=\frac{d_{y_{T}}}{2} \cdot \tan (q) \boldsymbol{\imath}-\frac{d_{y_{T}}}{2} \boldsymbol{\jmath} .
\end{aligned}
$$

Substituting (11) into (8) and with $\omega=\omega \mathbf{k}$, the expression for $v_{E}$ becomes

$$
\begin{aligned}
& \mathbf{v}_{E}=\mathbf{v}_{T}+\omega \mathbf{k} \times\left(\frac{d_{y_{T}}}{2} \cdot \tan (q) \boldsymbol{\imath}-\frac{d_{y_{T}}}{2} \boldsymbol{\jmath}\right), \\
& \mathbf{v}_{E}=\mathbf{v}_{T}+\frac{d_{y_{T}}}{2} \tan (q) \omega \boldsymbol{\jmath}+\frac{d_{y_{T}}}{2} \omega \boldsymbol{\imath} .
\end{aligned}
$$

Therefore the expressions for $v_{E_{x}}$ and $v_{E_{y}}$ to be used in (5), (6), and (7) are

$$
\begin{aligned}
& v_{E_{x}}=v_{T_{x}}+\frac{d_{y_{T}}}{2} \omega, \\
& v_{E_{y}}=v_{T_{y}}+\frac{d_{y_{T}}}{2} \tan (q) \omega .
\end{aligned}
$$

The term $A_{r}$ in (5) represents the reference area of the rigid body as represented in Figure 1. Because the impact in this model will be expressed in terms of the impact angle, then 
the reference area will change as the angle changes during impact. The expression for the reference area is

$$
\begin{aligned}
& A_{r}=d_{c} \frac{d_{y_{T}}}{\cos q}\left|\sin \left(q-q_{m}\right)\right|, \\
& q_{m}=\tan ^{-1}\left(\frac{v_{E_{x}}}{v_{E_{y}}}\right),
\end{aligned}
$$

where $q$ is the angle between the cylinder and the vertical axis and $q_{m}$ is the moving angle (see Figure 1) of the intruder. The volume $V$ of the immersed rigid body can also be calculated in terms of the impact angle:

$$
V=\frac{\pi d_{c}^{2}}{4} \frac{d_{y_{T}}}{\cos q} .
$$

Substituting (5)-(15) into (1) and separating the components of the vectors per their respective unit vector, the components of the resistance force are

$$
\begin{aligned}
& F_{d_{x}} \\
& =-\left(v_{T_{x}}+\frac{d_{y_{T}}}{2} \omega\right) \eta_{d} \rho_{g} d_{c} \frac{d_{y_{T}}}{\cos q} \\
& \cdot\left|\sin \left(q-\tan ^{-1}\left(\frac{v_{T_{x}}+\left(d_{y_{T}} / 2\right) \omega}{v_{T_{y}}+\left(d_{y_{T}} / 2\right) \tan (q) \omega}\right)\right)\right| \\
& \sqrt{\left(v_{T_{x}}+\frac{d_{y_{T}}}{2} \omega\right)^{2}+\left(v_{T_{y}}+\frac{d_{y_{T}}}{2} \tan (q) \omega\right)^{2}}, \\
& F_{d_{y}} \\
& =-\left(v_{T_{y}}+\frac{d_{y_{T}}}{2} \tan (q) \omega\right) \eta_{d} \rho_{g} d_{c} \frac{d_{y_{T}}}{\cos q} \\
& \cdot\left|\sin \left(q-\tan ^{-1}\left(\frac{v_{T_{x}}+\left(d_{y_{T}} / 2\right) \omega}{v_{T_{y}}+\left(d_{y_{T}} / 2\right) \tan (q) \omega}\right)\right)\right| \\
& \cdot \sqrt{\left(v_{T_{x}}+\frac{d_{y_{T}}}{2} \omega\right)^{2}+\left(v_{T_{y}}+\frac{d_{y_{T}}}{2} \tan (q) \omega\right)^{2}}, \\
& F_{s_{x}}=-\frac{\mathbf{v}_{E_{x}}}{\left|\mathbf{v}_{E_{x}}\right|} \eta_{h} g \rho_{g} d_{y_{T}}^{2} d_{c} \\
& F_{s_{y}}=-\frac{\mathbf{v}_{E_{y}}}{\left|\mathbf{v}_{E_{z}}\right|} \eta_{v}\left(\frac{d_{y_{T}}}{L}\right)^{\lambda} g \rho_{g} \frac{\pi d_{c}^{2}}{4} \frac{d_{y_{T}}}{\cos q} .
\end{aligned}
$$

The constants $\eta_{v}$ and $\lambda$ depend on the shape of the body, the container of the medium, the volume fraction of the granular matter, and the direction of motion of the penetrating object, that is, whether it is plunging in or withdrawing. These constants are expressed based on empirical data. These experiments $[3,15,23]$ focused specifically on the vertical impact of spheres at low speed $v_{0} \leq 5 \mathrm{~mm} / \mathrm{s}$, but it is also important that the medium used for the experiments in all three studies consisted of monodisperse glass beads. The recent work of Li et al. [14] has shown that the grain type has a considerable influence on the error obtained for their model. For natural sand, they observed an error that was $11 \%$ higher than the results obtained in glass beads.

The power law coefficient has been shown to be independent of both the impact speed and diameter of the intruder, but it does depend also on grain size and container size and hence the density of the medium [3]. For a cylinder intruder, $\lambda$ is smaller than 1.3 under the conditions described above for the plunging motion and greater than 1.7 for withdrawal $[15,23]$. The work of Bruyn and Walsh [10] has shown a clear dependency of the power law on grain size and found values for the power $\lambda$ that are less than 1 . The scaling coefficient for the vertical static force was measured [15] to be approximatively 10 for plunging and 0.5 for withdrawing motion, but other studies that used cylinders as probes for impacting a granular medium consisting of glass spheres [11] found the coefficient $\eta_{h}$ to be as high as 70, while Albert et al. [24] recorded values as low as 2.43 for cylinders of diameter up to $11.9 \mathrm{~mm}$. For the scaling of a drag force similar to the static vertical resistant force, Costantino et al. [29] observed a value for $\eta_{h}$ of approximatively 2.7.

In the recent work of Wang et al. [18], which utilized a force similar to the one used in this study, the authors concluded that all the scaling factors involved in the expression of the resistant force depend primarily on the angle of impact.

Since some of the studies mentioned that the scaling constants and power law in the resistance force, $\eta_{d}, \eta_{h}, \eta_{v}$, and $\lambda$, depend on the density of the media, and others demonstrated that these coefficients depend on the impact angle, it is now possible to propose a series of expressions that include both. In Table 1 we propose expressions for $\eta_{d}, \eta_{h}, \eta_{v}$, and $\lambda$ that have been developed empirically in terms of the numerical value, $\Lambda_{g}$, of the density of the granular matter, $\rho_{g}$, and the impact angle, $\alpha$. The expressions presented in Table 1 provide approximative values for the constants that can be implemented in the force model used in the simulations of a free link impact with granular matter.

Analyses of the dependency of the resistant force exerted by granular media onto a penetrating rigid body on the impact angle $[18,27]$ have clearly demonstrated the existence of a critical angle. However, for low impact angles the total resistance force involved in an impact with granular matter presents more complicated features.

\section{Experimental Setup and Results}

The schematic of the experimental system used to examine the impact of a kinematic chain with two impact points is shown in Figure 2. The two round ended steel links in this system were identical in mass and dimensions: diameter $d_{1}=$ $d_{2}=0.0064 \mathrm{~m}$ and length $L_{1}=L_{2}=0.135 \mathrm{~m}$. Each link had a plastic washer with a thickness of $0.5 \mathrm{~mm}$ around the screw hole. This washer was placed between the links to reduce the friction between the two links to a negligible level while at the same time allowing them to rotate around a screw at the top. $H$ is the height with respect to which the initial 
TABLE 1: The analytical expressions for the constants $\eta_{d}, \eta_{h}, \eta_{v}$, and $\lambda$ in the resistance force formulation.

\begin{tabular}{lcccc}
\hline Angle $\alpha=\pi / 2-q$ & $\eta_{h}$ & $\eta_{d}$ & $\eta_{v}$ & $\lambda$ \\
\hline 90 & $\frac{\Lambda_{g}}{\alpha^{2} 100}$ & $\frac{3}{2} \eta_{h} \alpha$ & $10 \eta_{h} \alpha^{3}$ & $\frac{\Lambda_{g}}{\alpha} \cdot 10^{-4}$ \\
\hline 45 & $\frac{\Lambda_{g} \alpha^{2}}{100}$ & $\frac{2}{3} \eta_{h} q$ & $10 \eta_{h} \alpha^{3}$ & $2 \Lambda_{g} \alpha \cdot 10^{-4}$ \\
\hline 15 & $\frac{\Lambda_{g} \alpha^{2}}{10}$ & $\frac{2}{3} \eta_{h} \alpha$ & $10 \eta_{h} q^{3}$ & $2 \Lambda_{g} q \cdot 10^{-4}$ \\
\hline
\end{tabular}

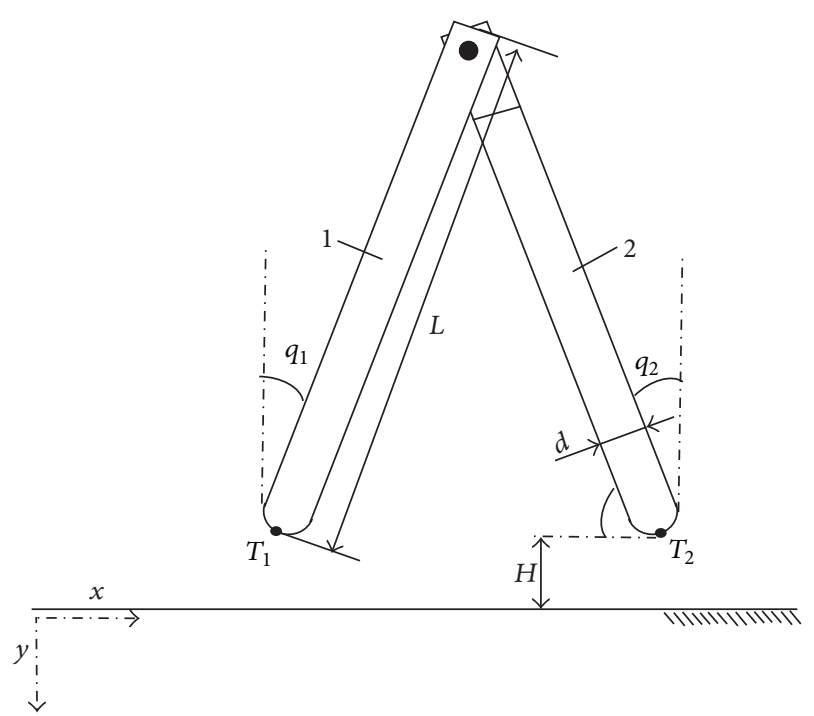

FIGURE 2: Two-link kinematic chain, for which Link 1 makes an angle $q_{1}$ with vertical and Link 2 makes and angle $q_{2}$ with vertical.

impact velocity $v_{0}$ is defined. For all the models in this study, the impact is defined from the moment the tip of the bar $T$ contacts the granular surface ( $v_{T}$ is maximum) and ends when the vertical component of the velocity of the point $T$ is zero. The penetration depth, $d_{y_{T}}$, is the distance between the surface of the granular bed and the end tip of the cylinder. The initial conditions are given by the initial impact angle $q$ and initial impact velocity $v_{0}$. Angle $q$ is the angle of the bar with the vertical axis $y$, and $\alpha=\pi / 2-q$.

The motion capture system used for this study is the Northern Digital Inc., OPTOTRAK 3020. The system consists of IR markers, a strober, position sensors (IR cameras), a Control Unit, and a PC (Figure 3). The OPTOTRAK 3020 can track up to 256 inferred markers connected through the strober to the control unit. The control unit is connected to the position sensors to measure changes in the position of the markers with time. The user has access to the data recorded through the NDI ToolBench software, which also controls the system hardware.

Infrared markers are attached to the chain system. One marker is positioned in the center of the mass of the link, one marker, $M_{2}$, is at the top joint, and a third marker is located on the surface of the granular matter for reference. The motion capture system in Figure 3 records the position of the markers with an RMS accuracy of $0.1 \mathrm{~mm}$ [30] and

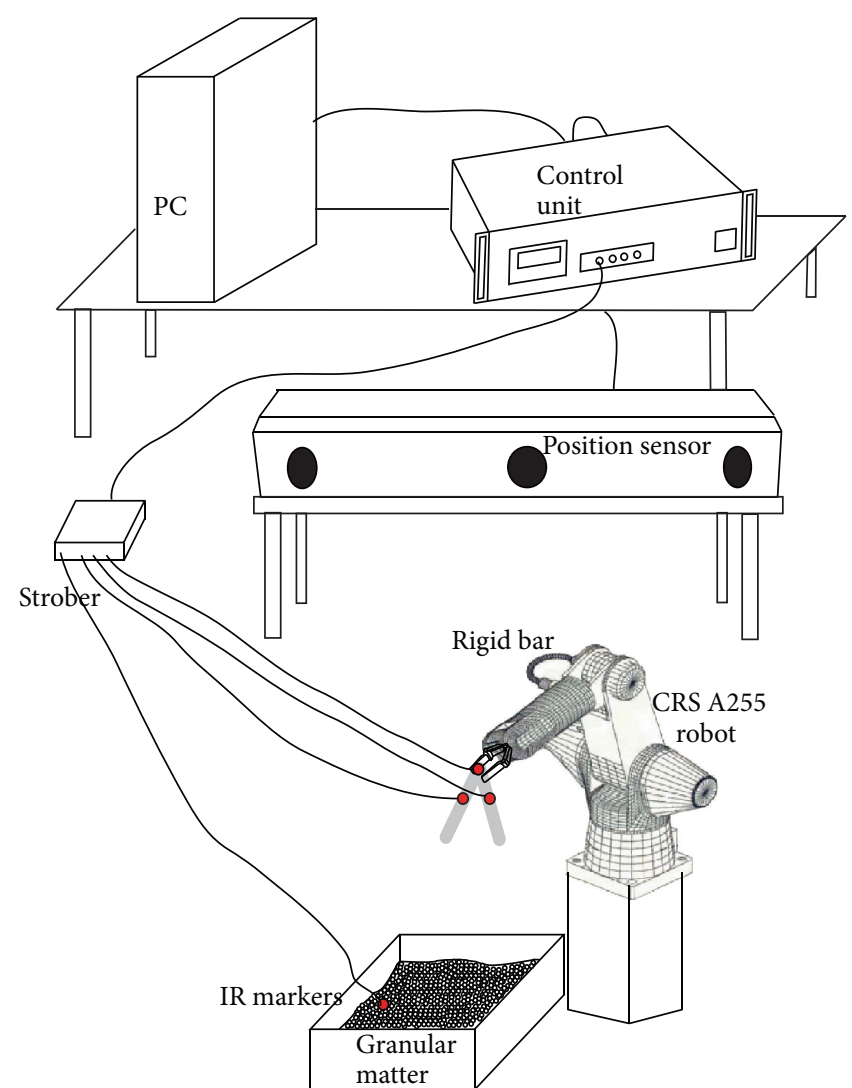

FIGURE 3: OPTOTRAK 3020 equipment setup used for the experimental study of the impact of a two-link kinematic chain with two points of impact with granular matter.

does not require calibration. The 3D position in time of the two markers attached to the rigid free link is captured during the free fall at a sample rate of 650 frames/s. The granular medium utilized for the experiments in this study was "Play Sand" (Quikrete 1113-51). This kind of sand was chosen over glass beads or monodisperse beads due to the fact that its properties are more appropriate for applications involving multilegged robots that must move across many different types of terrain. Li et al. [14] performed tests on dry granular media with various particle sizes and properties. They reported large errors between tests on natural sand and glass beads and poppy seeds. For the purpose of this study, natural polydisperse grains represent the optimum choice. A box of dimensions $0.45 \times 0.32 \times 0.09 \mathrm{~m}$ (length $\times$ width $\times$ height) was filled with the granular matter. The dimensions of the box were sufficiently larger than the intruder to ensure that no Jansen effect would occur. In order to preserve the integrity of the results for each data collection, the sand was stirred and leveled before each drop of the system. This procedure was utilized to maintain volume fraction and eliminate history effects.

Using the setup in Figure 3 the impact with granular matter was inspected under several initial conditions. The impact angle $q$ is defined relative to the upward vertical axis. The initial velocity was defined as the linear velocity of the end 
TABLE 2: The experimental results for the two end points of the double kinematic chain.

\begin{tabular}{lccc}
\hline & $t_{s}(\mathrm{~s})$ & $d_{y_{T}}(\mathrm{~m})$ & $v_{y_{T}}(0)(\mathrm{m} / \mathrm{s})$ \\
\hline \multirow{3}{*}{ Link 1 } & $0.025 \pm 0.003$ & $0.035 \pm 0.002$ & $2.58 \pm 0.021$ \\
& $0.026 \pm 0.003$ & $0.030 \pm 0.001$ & $2.29 \pm 0.118$ \\
& $0.029 \pm 0.001$ & $0.025 \pm 0.0005$ & $1.60 \pm 0.036$ \\
\hline \multirow{3}{*}{ Link 2 } & $0.026 \pm 0.002$ & $0.037 \pm 0.001$ & $2.57 \pm 0.036$ \\
& $0.030 \pm 0.005$ & $0.031 \pm 0.002$ & $2.3 \pm 0.095$ \\
& $0.032 \pm 0.002$ & $0.025 \pm 0.0003$ & $1.61 \pm 0.025$ \\
\hline
\end{tabular}

of the bar $T, v_{y_{T}}$. Three cases were considered: for $v 1_{01}(0)=$ $2.62 \mathrm{~m} / \mathrm{s}, v 2_{01}(0)=2.62 \mathrm{~m} / \mathrm{s}$; for $v 1_{02}(0)=2.30 \mathrm{~m} / \mathrm{s}$, $v 2_{02}(0)=2.30 \mathrm{~m} / \mathrm{s}$; for $v 1_{03}(0)=1.60 \mathrm{~m} / \mathrm{s}, v 2_{03}(0)=$ $1.60 \mathrm{~m} / \mathrm{s}$. For each case, five recordings were analyzed. The impact data extracted is the displacement for the end point $T$ of the free link. The impact starts at $d_{y_{T}}(0)=0$ and the end of the penetration phase is taken to be the time at which the linear vertical velocity of the tip of the bar is zero, $v_{y_{T}}(t)=0$. The stopping time represents the time it takes the link to burrow into the granular matter volume up to the point when $v_{y_{T}}\left(t_{s}\right)=0$. Table 2 shows the recorded stopping time, $t_{s}$; final displacement, $d_{y_{T}}\left(t_{s}\right)$; the initial impact velocities chosen, $v_{y_{T}}$.

The experimental results show that increasing of the initial impact velocity leads to a decrease in stopping time. This means that, for lower impact velocities, it takes longer for the penetrating object to stop moving, even though the distance traveled is shorter.

\section{Results}

The two-link kinematic chain is modeled in SolidWorks according to the specifics of the experimental design. The resistance force used for each impact point of the system has the formulation given in (16). For the simulation of the resistant force, $F_{r_{i}}, i=1,2$, the values of the four constants were estimated using Table 3 . The calculated values that were substituted for the coefficients in the $F_{r_{i}}$ expression are given in Table 4.

The motion analysis was run for three initial velocities and the angles of the two links with the vertical were the same as in the experimental setup. The colored curves in Figure 4 show the simulation results for the immersion of the tip point of Link 1, Figure 4(a), and Link 2, Figure 4(b), conditioned by the initial velocity.

For each link, the phenomenon normally associated with a low velocity impact with granular matter appeared. The final displacement corresponding to zero value for the vertical linear velocity of the end point $T_{i}$ increased with initial velocity, but the stopping time for both links decreased with increasing initial velocity. The displacement in time for each link during simulations was very similar to the average displacement in time observed in the experimental trials (black curves in Figure 4). The relative error between the simulation results and the experimental final displacement is shown in Table 5. The two-link impact with the granular
TABLE 3: The expressions for resistance force coefficients $\eta_{h}, \eta_{d}, \eta_{v}$, and $\lambda$.

\begin{tabular}{cccccc}
\hline & $\begin{array}{r}v_{T_{y}}(0) \\
(\mathrm{m} / \mathrm{s})\end{array}$ & $\eta_{h}$ & $\eta_{d}$ & $\eta_{v}$ & $\lambda$ \\
\hline Link 1 & 2.60 & $0.6 \cdot \frac{\Lambda_{g} \alpha_{1}^{2}}{10}$ & $\frac{3}{2} \eta_{h} \alpha_{1}$ & $1.55 \eta_{h} \alpha_{1}^{3}$ & $\frac{\Lambda_{g}}{\alpha_{1}} \cdot 10^{-4}$ \\
& 2.29 & $\frac{\Lambda_{g} \alpha_{1}^{2}}{10}$ & $\frac{3}{2} \eta_{h} \alpha_{1}$ & $1.55 \eta_{h} \alpha_{1}^{3}$ & $\frac{\Lambda_{g}}{\alpha_{1}} \cdot 10^{-4}$ \\
& 1.60 & $\frac{\Lambda_{g} \alpha_{1}^{2}}{10}$ & $\frac{3}{2} \eta_{h} \alpha_{1}$ & $1.55 \eta_{h} \alpha_{1}^{3}$ & $\frac{\Lambda_{g}}{\alpha_{1}} \cdot 10^{-4}$ \\
\hline \multirow{2}{*}{2.60} & $1.25 \cdot \frac{\Lambda_{g}}{\alpha_{2}^{2} \cdot 100}$ & $\frac{2}{3} \eta_{h} \alpha_{2}$ & $5 \eta_{h} \alpha_{2}^{3}$ & $\Lambda_{g} \alpha_{2} \cdot 10^{-4}$ \\
& 2.30 & $1.25 \cdot \frac{\Lambda_{g}}{\alpha_{2}^{2} 100}$ & $\frac{2}{3} \eta_{h} \alpha_{2}$ & $5.25 \eta_{h} \alpha_{2}^{3}$ & $\Lambda_{g} \alpha_{2} \cdot 10^{-4}$ \\
& 1.61 & $1.25 \cdot \frac{\Lambda_{g}}{\alpha_{2}^{2} 100}$ & $\frac{2}{3} \eta_{h} \alpha_{2}$ & $5.25 \eta_{h} \alpha_{2}^{3}$ & $\Lambda_{g} \alpha_{2} \cdot 10^{-4}$ \\
\hline
\end{tabular}

TABLE 4: The values for the resistance force coefficients $\eta_{h}, \eta_{d}, \eta_{v}$, and $\lambda$.

\begin{tabular}{lccccc}
\hline & $v_{T_{y}}(0)(\mathrm{m} / \mathrm{s})$ & $\eta_{h}$ & $\eta_{d}$ & $\eta_{v}$ & $\lambda$ \\
\hline \multirow{3}{*}{ Link 1 } & 2.60 & 8 & 13 & 33 & 0.125 \\
& 2.29 & 9 & 19 & 33 & 0.13 \\
& 1.60 & 10 & 19 & 38 & 0.14 \\
\hline \multirow{3}{*}{ Link 2 } & 2.60 & 11.8 & 10 & 137 & 0.205 \\
& 2.30 & 12.5 & 10.8 & 144 & 0.21 \\
& 1.61 & 13 & 12 & 145 & 0.22 \\
\hline
\end{tabular}

TABLE 5: Relative error between the experimental and simulation results for penetration depth.

\begin{tabular}{lcc}
\hline & $v_{y_{T}}(0)(\mathrm{m} / \mathrm{s})$ & $\epsilon_{i}(\%)$ \\
\hline \multirow{3}{*}{ 1 for Link 1 } & 2.62 & 1.77 \\
& 2.29 & 0.61 \\
& 1.60 & 1.31 \\
\hline \multirow{3}{*}{ 2 for Link 2 } & 2.60 & 1.77 \\
& 2.29 & 3.72 \\
& 1.61 & 2.39 \\
\hline
\end{tabular}

surface is analyzed here for the angles $q_{1}=-15^{\circ}$ and $q_{2}=15^{\circ}$. For angles $q_{1}<-75^{\circ}$ and $q_{2}>85^{\circ}$ the features of the impact will become more complicated, but the phenomenon characteristic to the penetration of the granular matter should appear still.

The total resistance force $F_{r_{i}}=\sqrt{\left(F_{s_{x_{i}}}+F_{d_{x_{i}}}\right)^{2}+\left(F_{s_{x_{i}}}+F_{d_{x_{i}}}\right)^{2}}$ on each link is presented in Figure 5. The resistance force increases with increasing initial velocity for each link. During the penetration, the resistance force will increase according to the dynamic force until this component reaches its maximum and then the resistance force will act more and more under the influence of the static force. The penetration phase ends with the resistance force having a magnitude equal to the static force. 


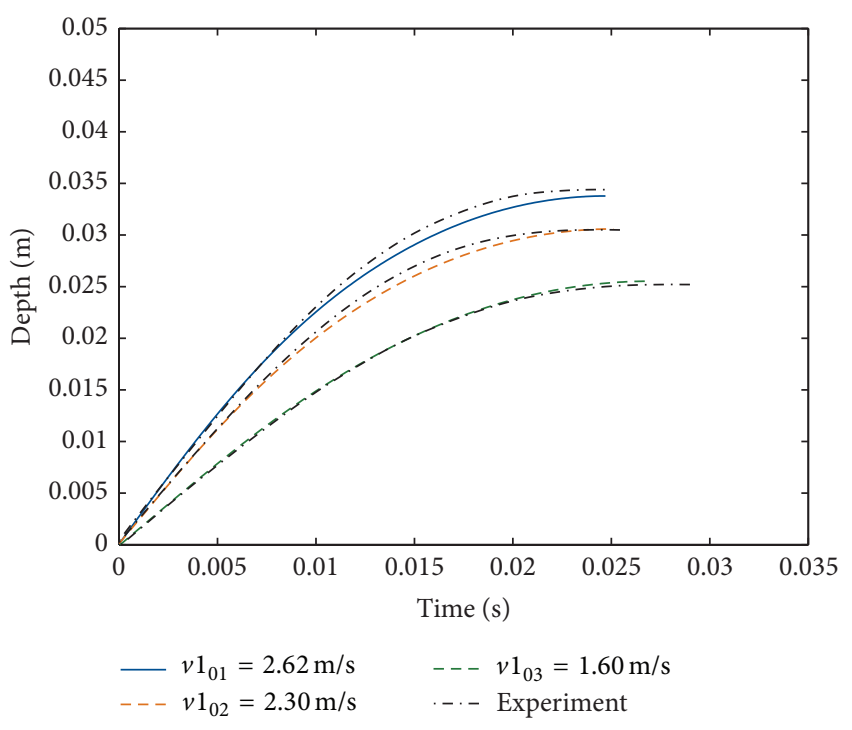

(a) Displacement in time of the tip point $T_{1}$ for Link 1

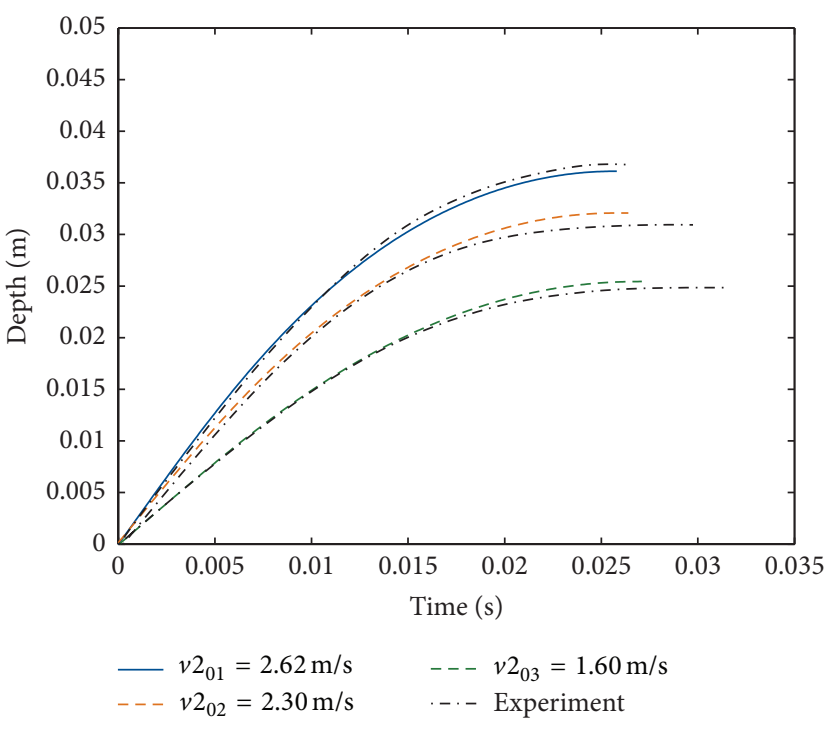

(b) Displacement in time of the tip point $T_{2}$ for Link 2

FIGURE 4: Simulation and experimental impact results for the penetration displacement of a double kinematic chain with two contact points: (a) for Link 1 and (b) for Link 2 .

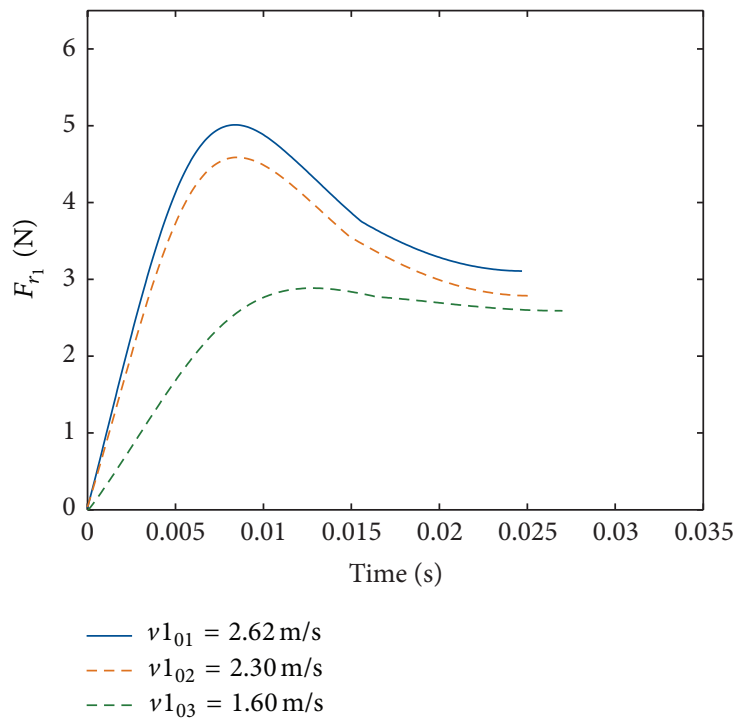

(a) The reaction of the resistance force on Link 1

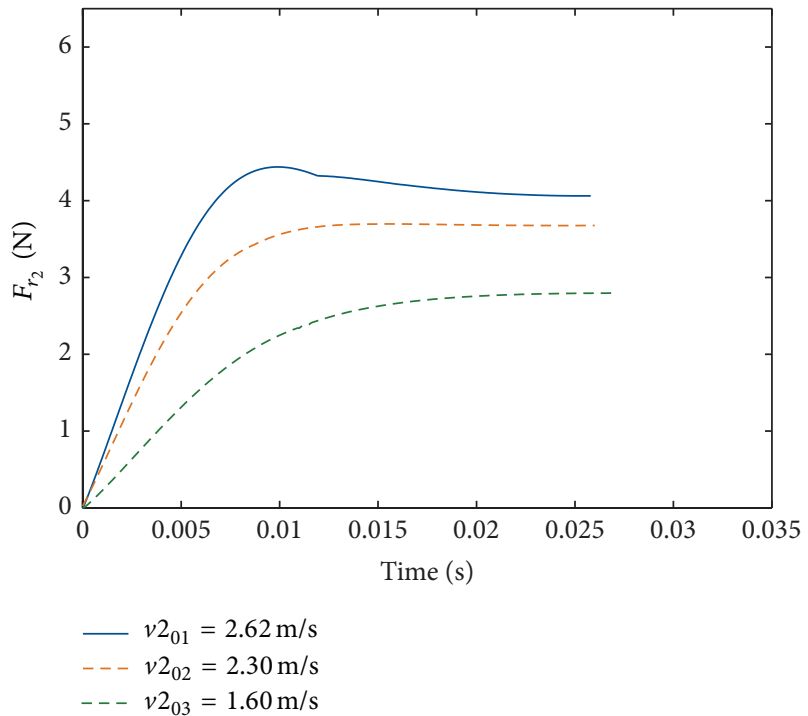

(b) The reaction of the resistance force on Link 2

FIGURE 5: The resistance force during penetration phase on the impacting system: (a) for Link 1 with impact angle $q_{1}=-15^{\circ}$, and (b) for Link 2 with impact angle $q_{2}=15^{\circ}$.

Figure 5 shows that the resistance force depends on the dynamic force longer at higher initial velocities. This is also depicted in Figure 6, which shows the instant at which the dynamic and static components interchange influences. For Link 2, the dependence of the resistance force on the dynamic force with initial velocity is more pronounced (Figure 6(b)) and the dynamic force will exert an influence on the resistance force for longer than at higher velocities. This is reversed at low velocities, where the displacement dependency will dominate longer. For Link 1 (Figure 6(a)), the static force will prevail for longer than at higher velocities because, for this link, the magnitudes of the dynamic force for $v 1_{01}$ and $v 1_{02}$ are so close in value and in appearance in time that the velocity dependence and displacement velocity will switch dominance almost at the same time.

The total energy dissipated to the granular medium by each link $\Delta E_{i}=\Delta$ K.E. $_{i}+\Delta{\mathrm{P} . E_{i}}_{i}$ is shown in Figure 7. The datum for the potential energy is considered the surface of the granular media. The error between the two links in total energy dissipated stabilizes to $0.6 \%$ for all three initial 


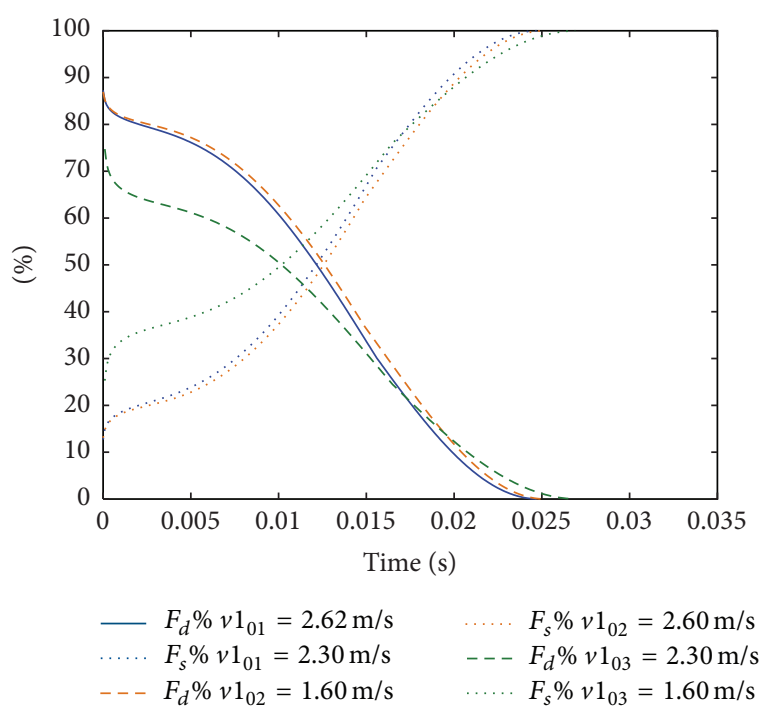

(a) The contribution of the dynamic and static reaction on Link 1

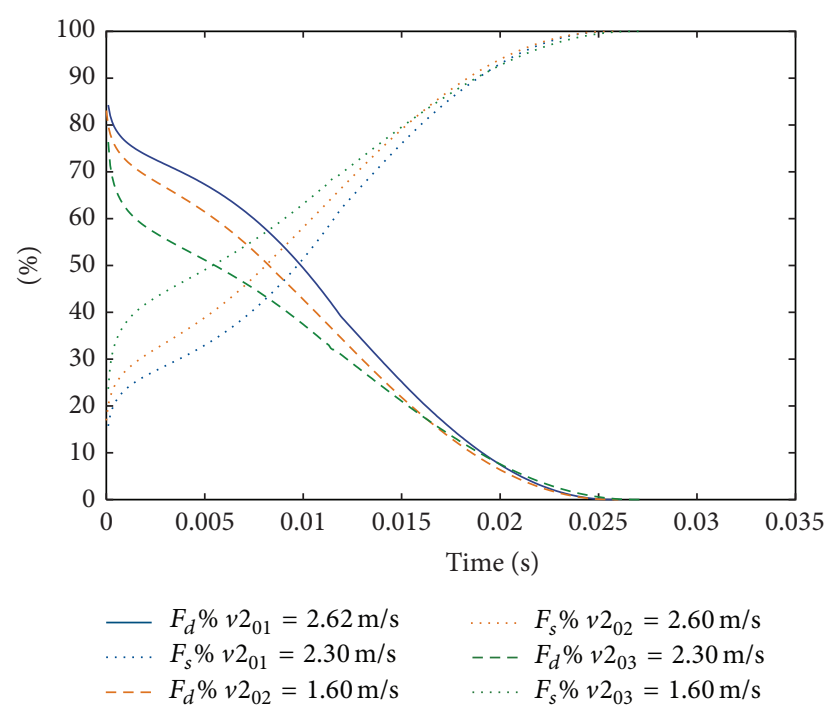

(b) The contribution of dynamic and static reaction on Link 2

FIGURE 6: Dynamic and static force percent out of the resistance force for an impact of a two-link kinematic chain with granular matter during the penetration phase: (a) for Link 1 and (b) for Link 2.

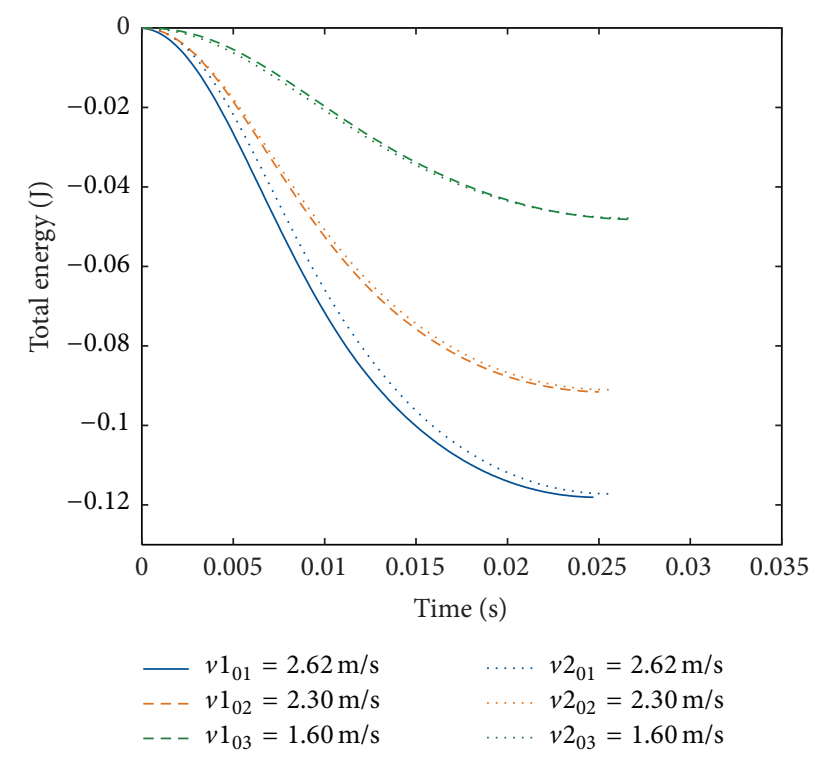

Figure 7: The total mechanical energy dissipated to the granular environment during penetration phase.

velocities. The total energy released to the medium increases with initial velocity $\left(\Delta E=0.24 \mathrm{~J}\right.$ at $v_{01}=2.6 \mathrm{~m} / \mathrm{s}, \Delta E=0.18 \mathrm{~J}$ at $v_{02}=2.3 \mathrm{~m} / \mathrm{s}$, and $\Delta E=0.10 \mathrm{~J}$ at $v_{03}=1.6 \mathrm{~m} / \mathrm{s}$ ).

The kinetic energy starts with a concave curvature but then goes through an inflection point and changes curvature. Interestingly, the potential energy does not show the same tendency and so will have an initial influence on the total energy values. However, due to the approximatively 10 times higher values for the kinetic energy, the potential energy will present a logarithmic like influence on the total energy while the kinetic energy will increase and come to dominate the energy dissipation trend (Figure 8). The lower the velocity, the longer it will take for the percentage of kinetic energy to increase.

\section{Conclusions}

For the impact of a two-link kinematic system with granular matter, the experiments showed that the system will stop faster with increasing initial velocity but will immerse deeper into the volume. The analysis was performed for each link separately, each with its own contact point. The experimental system settings were utilized for the simulations and a model was created in SolidWorks. The series of resistance force components, both for quadratic velocity and for the linear displacement-dependent variables, were used to simulate the impact with granular matter. Appropriate force coefficients $\eta_{h}, \eta_{d}, \eta_{v}$, and $\lambda$ expressions and values were proposed. The errors between the simulation and experimental results were low, and the behavior was consistent. The simulations also revealed the impact of the dynamic and static components on the resistance force and the percentage contribution of each. For increasing velocity, a slower shift in dominance from dynamic force to static force was both expected and demonstrated during the simulations. The resulting curves describe the progression of the dynamic and static forces and, in consequence of the resistance force, are consistent for each link when compared to the forces obtained for the single link impact angle model at the impact angles used in this study. The total mechanical energy for each link is preponderantly due to the kinetic energy for higher velocities, with the potential energy of the system released to the environment about being at the most 10 times smaller than the kinetic energy. Each link contributes about $50 \%$ to the total mechanical energy discharged to the environment for the system consisting of two identical links positioned at the same angle with the vertical. 


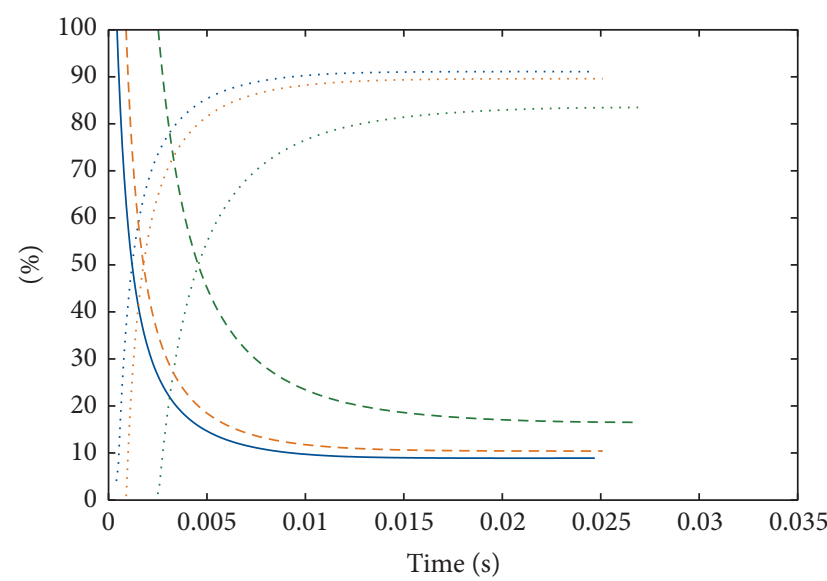

$$
\begin{aligned}
& \ldots . \text { K.E. } \% v 1_{01}=2.62 \mathrm{~m} / \mathrm{s}-\text { P.E. } \% v 1_{01}=2.62 \mathrm{~m} / \mathrm{s} \\
& \ldots \text { K.E. } \% v 1_{02}=2.30 \mathrm{~m} / \mathrm{s}-- \text { P.E. } \% v 1_{02}=2.30 \mathrm{~m} / \mathrm{s} \\
& \ldots . \text { K.E. } \% v 1_{03}=1.60 \mathrm{~m} / \mathrm{s}-- \text { P.E. } \% v 1_{03}=1.60 \mathrm{~m} / \mathrm{s}
\end{aligned}
$$

(a) The percentage of kinetic and potential energy that accounts for the total energy dissipation for Link 1

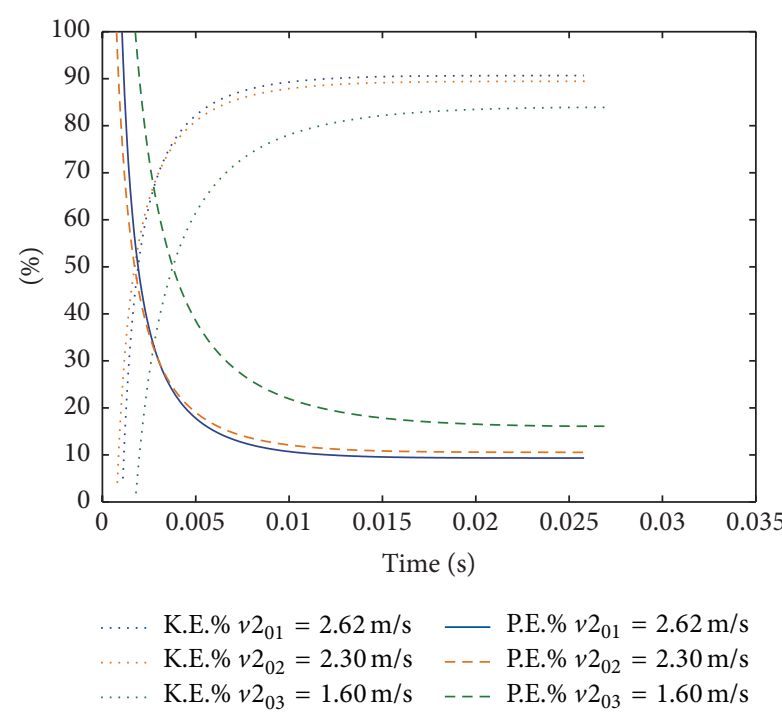

(b) The percentage of kinetic and potential energy that accounts for the total energy dissipation for Link 2

FIGURE 8: The contribution of the kinetic and potential energy to the total energy dissipation during the penetration phase by the system to the environment: (a) for Link 1 and (b) for Link 2.

\section{Conflict of Interests}

The authors declare that there is no conflict of interests regarding the publication of this paper.

\section{References}

[1] T. Faug, P. Caccamo, and B. Chanut, "Equation for the force experienced by a wall overflowed by a granular avalanche: experimental verification," Physical Review E, vol. 84, no. 5, Article ID 051301, 7 pages, 2011.

[2] A. Seguin, Y. Bertho, P. Gondret, and J. Crassous, "Dense granular flow around a penetrating object: experiment and hydrodynamic model," Physical Review Letters, vol. 107, no. 4, Article ID 048001, 2011.

[3] K. Matsuyama and H. Katsuragi, "Power law statistics of force and acoustic emission from a slowly penetrated granular bed," Nonlinear Processes in Geophysics, vol. 21, no. 1, pp. 1-8, 2014.

[4] H. Katsuragi and D. J. Durian, "Unified force law for granular impact cratering," Nature Physics, vol. 3, no. 6, pp. 420-423, 2007.

[5] L. S. Tsimring and D. Volfson, "Modeling of impact cratering in granular media," in Powders and Grains 2005, pp. 1215-1223, 2005.

[6] J. S. Uehara, M. A. Ambroso, R. P. Ojha, and D. J. Durian, "Lowspeed impact craters in loose granular media," Physical Review Letters, vol. 90, no. 19, Article ID 194301, 4 pages, 2003.

[7] F. Guillard, Y. Forterre, and O. Pouliquen, "Depth-independent drag force induced by stirring in granular media," Physical Review Letters, vol. 110, no. 13, Article ID 138303, 5 pages, 2013.

[8] F. Guillard, Y. Forterre, and O. Pouliquen, "Lift forces in granular media," Physics of Fluids, vol. 26, no. 4, Article ID 043301, 2014.
[9] R. Albert, M. A. Pfeifer, A.-L. Barabási, and P. Schiffer, "Slow drag in a granular medium," Physical Review Letters, vol. 82, no. 1, pp. 205-208, 1999.

[10] J. R. Bruyn and A. M. Walsh, "Penetration of spheres into loose granular media," Canadian Journal of Physics, vol. 82, no. 6, pp. 439-446, 2004.

[11] T. A. Brzinski III, P. Mayor, and D. J. Durian, "Depth-dependent resistance of granular media to vertical penetration," Physical Review Letters, vol. 111, Article ID 168002, 2013.

[12] Y. Ding, N. Gravish, and D. I. Goldman, "Drag induced lift in granular media," Physical Review Letters, vol. 106, no. 2, Article ID 028001, 2011.

[13] C. Li, S. T. Hsieh, and D. I. Goldman, "Multi-functional foot use during running in the zebra-tailed lizard (Callisaurus draconoides)," The Journal of Experimental Biology, vol. 215, no. 18, pp. 3293-3308, 2012.

[14] C. Li, T. Zhang, and D. I. Goldman, "A terradynamics of legged locomotion on granular media," The European Physical Journal, vol. 35, no. 7, pp. 1-12, 2013.

[15] G. Hill, S. Yeung, and S. A. Koehler, "Scaling vertical drag forces in granular media," Europhysics Letters, vol. 72, no. 1, pp. 137-143, 2005.

[16] D. I. Goldman and P. Umbanhowar, "Scaling and dynamics of sphere and disk impact into granular media," Physical Review E, vol. 77, no. 2, Article ID 021308, 14 pages, 2008.

[17] N. Gravish, P. B. Umbanhowar, and D. I. Goldman, "Force and flow at the onset of drag in plowed granular media," Physical Review E-Statistical, Nonlinear, and Soft Matter Physics, vol. 89, no. 4, Article ID 042202, 2014.

[18] D. Wang, X. Ye, and X. Zheng, "The scaling and dynamics of a projectile obliquely impacting a granular medium," The European Physical Journal E, vol. 35, no. 7, pp. 1-12, 2012.

[19] M. A. Ambroso, R. D. Kamien, and D. J. Durian, "Dynamics of shallow impact cratering," Physical Review E, vol. 72, no. 4, Article ID 041305, 4 pages, 2005. 
[20] M. Hou, Z. Peng, R. Liu, K. Lu, and C. K. Chan, "Dynamics of a projectile penetrating in granular systems," Physical Review E, vol. 72, no. 6, Article ID 062301, 2005.

[21] S. Lee and D. B. Marghitu, "Analysis of a rigid body obliquely impacting granular matter," Nonlinear Dynamics, vol. 57, no. 12, pp. 289-301, 2009.

[22] N. Mazouchova, N. Gravish, A. Savu, and D. I. Goldman, "Utilization of granular solidification during terrestrial locomotion of hatchling sea turtles," Biology Letters, vol. 6, no. 3, pp. 398401, 2010.

[23] Z. Peng, X. Xu, K. Lu, and M. Hou, "Depth dependence of vertical plunging force in granular medium," Physical Review E-Statistical, Nonlinear, and Soft Matter Physics, vol. 80, no. 2, Article ID 021301, 2009.

[24] I. Albert, J. G. Sample, A. J. Morss, S. Rajagopalan, A.-L. Barabási, and P. Schiffer, "Granular drag on a discrete object: shape effects on jamming," Physical Review E, vol. 64, no. 6, Article ID 061303, 2001.

[25] P. Umbanhowar and D. I. Goldman, "Granular impact and the critical packing state," Physical Review E, vol. 82, no. 1, Article ID 010301, 4 pages, 2010.

[26] D. B. Marghitu and S. Lee, "Analytical and experimental analysis of a free link in contact with a granular medium," The Scientific World Journal, vol. 2013, Article ID 808574, 9 pages, 2013.

[27] C. Li, P. B. Umbanhowar, H. Komsuoglu, and D. I. Goldman, "The effect of limb kinematics on the speed of a legged robot on granular media," Experimental Mechanics, vol. 50, no. 9, pp. 1383-1393, 2010.

[28] T. Faug, R. Beguin, and B. Chanut, "Mean steady granular force on a wall overflowed by free-surface gravity-driven dense flows," Physical Review E, vol. 80, no. 2, Article ID 021305, 13 pages, 2009.

[29] D. J. Costantino, J. Bartell, K. Scheidler, and P. Schiffer, "Lowvelocity granular drag in reduced gravity," Physical Review E, vol. 83, no. 1, Article ID 011305, 2011.

[30] Optotrack brochure, http://www.ndigital.com/. 

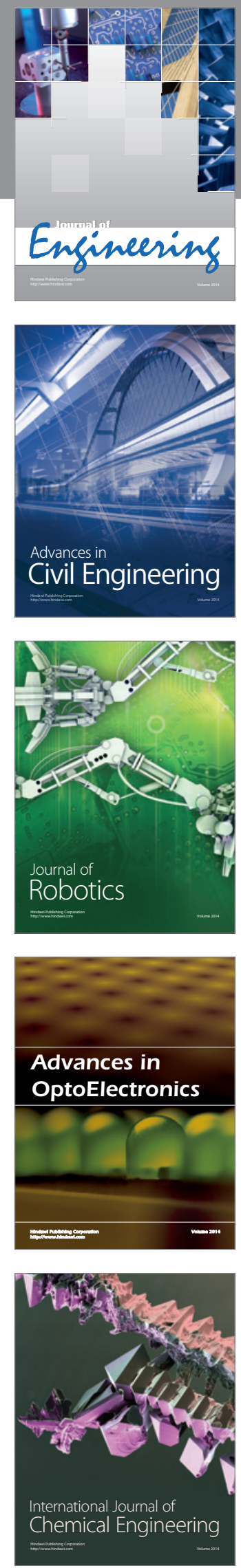

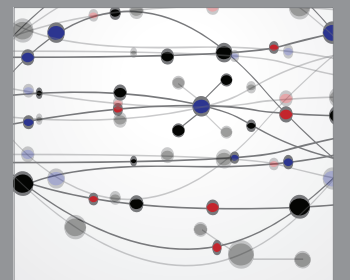

The Scientific World Journal
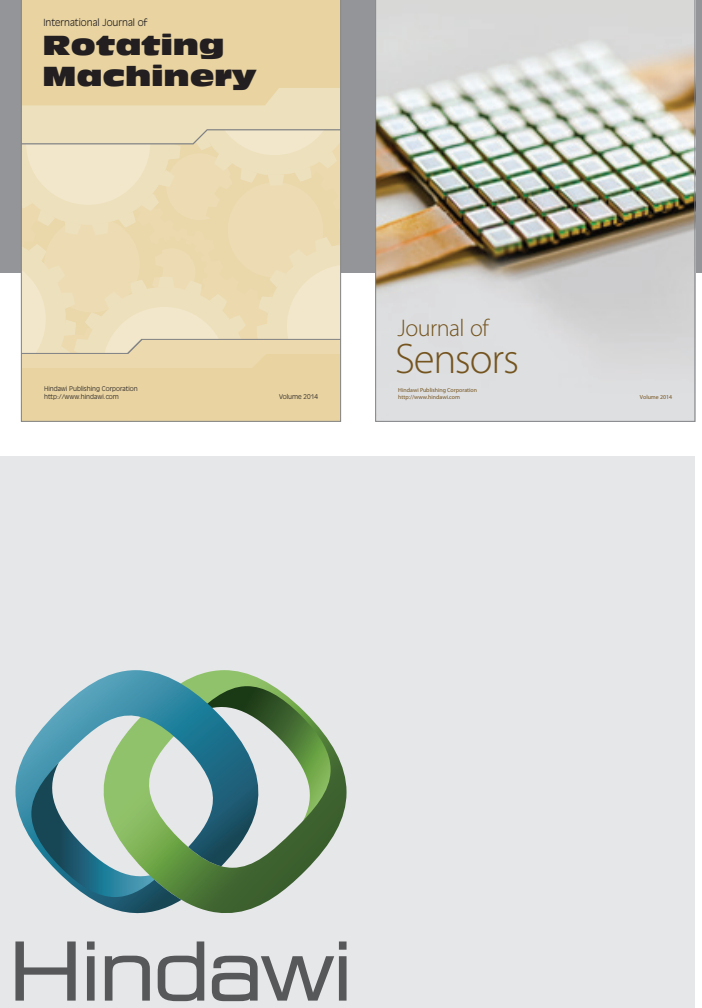

Submit your manuscripts at http://www.hindawi.com
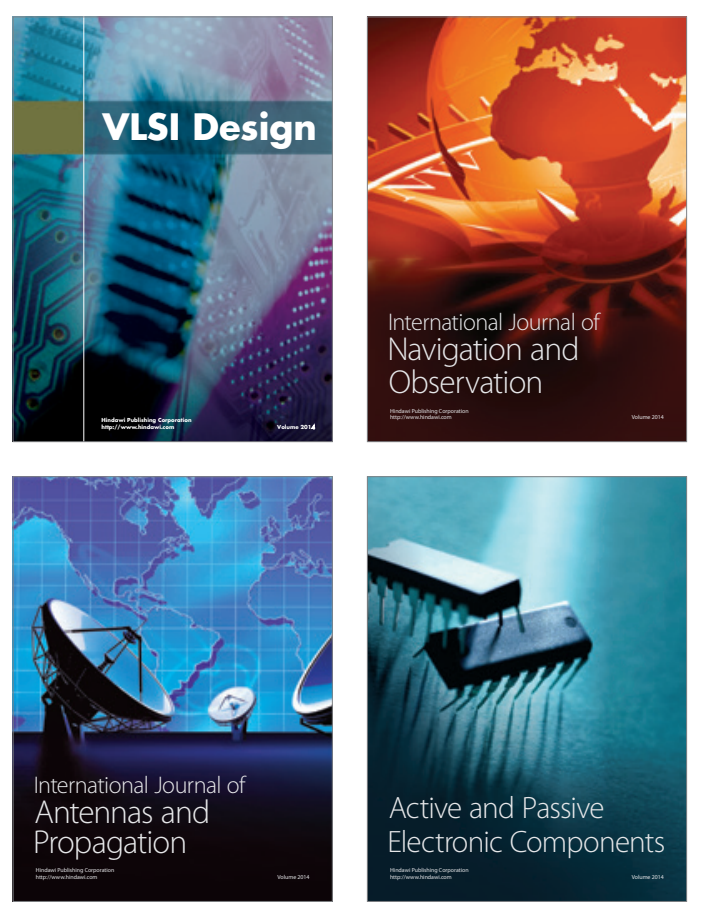
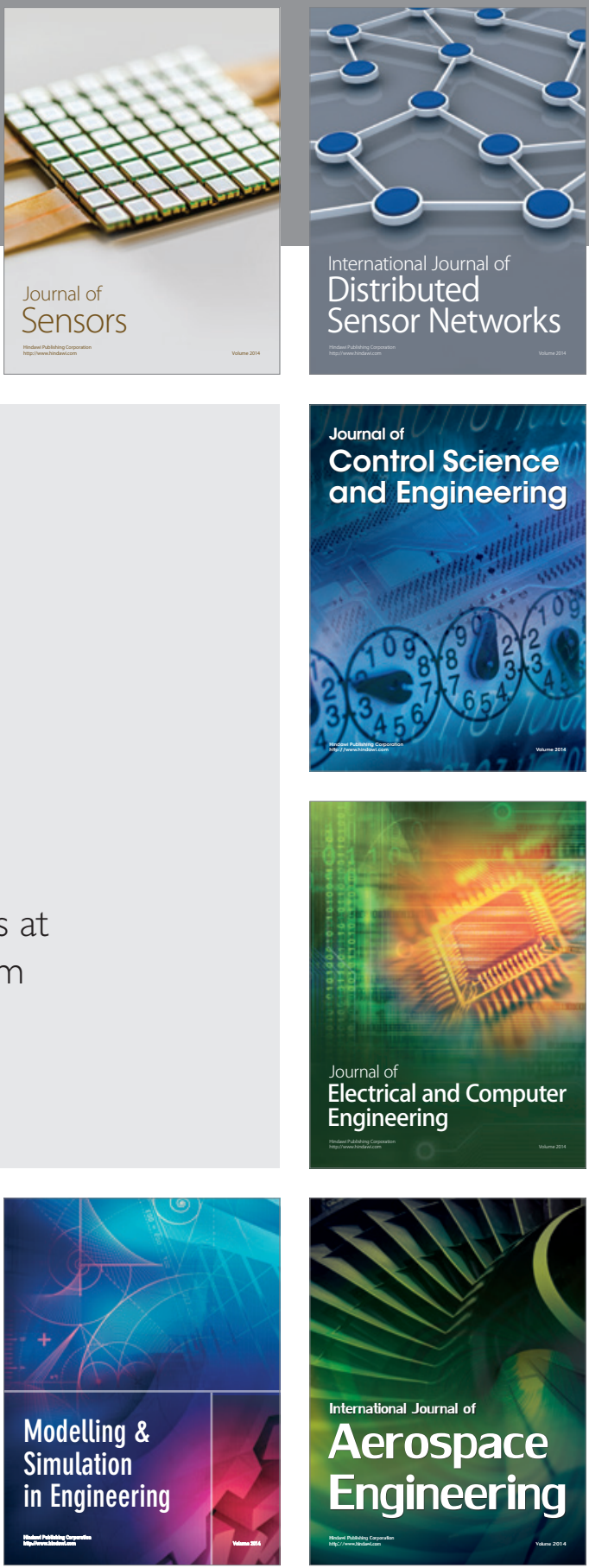

Journal of

Control Science

and Engineering
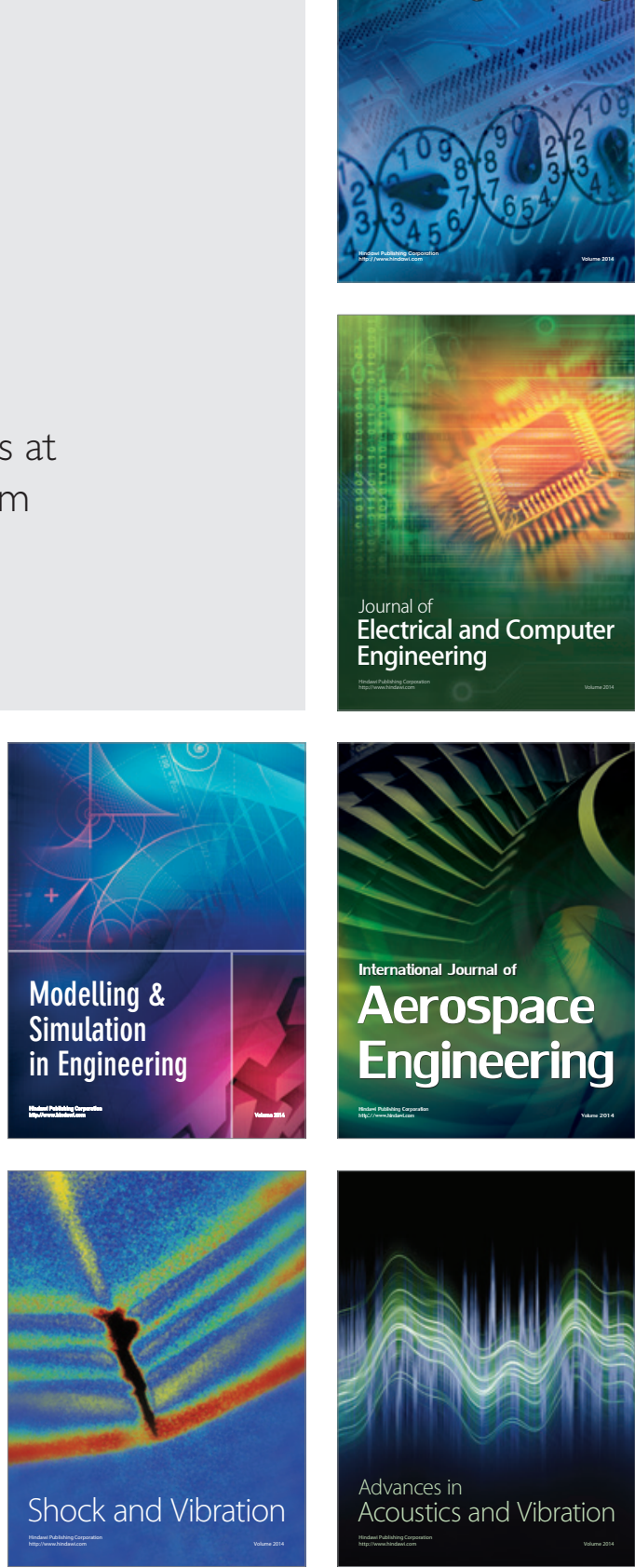